\title{
La implementación de las redes sociales en el sector hoteleiro español
}

The implementation of online social networks in the Spanish hotel sector

\author{
Alfonso Infante-Moro \\ Grupo de Investigación GITICE, Universidad de Huelva, España \\ alfonso.infante@uhu.es \\ Juan-Carlos Infante-Moro \\ Grupo de Investigación GITICE, Universidad de Huelva, España \\ juancarlos.infante@uhu.es \\ Francisco-José Martínez-López \\ Grupo de Investigación GITICE, Universidad de Huelva, España \\ francis@uhu.es \\ Mercedes García-Ordaz \\ Grupo de Investigación GITICE, Universidad de Huelva, España \\ ordaz@uhu.es \\ Julia Gallardo-Pérez \\ Grupo de Investigación GITICE, Universidad de Huelva, España \\ julia.gallardo@uhu.es
}

\begin{abstract}
Resumen
Este estudio nos describe cómo ha sido el proceso de implementación de las redes sociales online generalizadas (Facebook, Twitter... ) en el sector hotelero, analizando dicho fenómeno a través de encuesta y utilizando como marco comparativo el sector empresarial español en general. Como resultado, hemos obtenido que este fenómeno no ha tenido la misma acogida en el sector empresarial español que en el sector hotelero, ya que la diferencia entre el número de hoteles en España que tienen presencia en estas redes sociales respecto al número de empresas del resto del sector empresarial español es bastante amplia. Lo que se traduce en la mayor importancia que da este sector hotelero a estas herramientas respecto al resto del sector empresarial español.
\end{abstract}

Palabras-clave: Hoteles, empresas, redes sociales, implementación, internet, TIC.

\begin{abstract}
This study describes the process of implementation of generalized online social networks (e.g. Facebook, Twitter) in the hotel sector, analyzing this phenomenon through a survey and using the Spanish business sector in general as a comparative framework. We found that this phenomenon has not had the same reception in the Spanish business setor as in the hotel sector, since the difference between the number of hotels in Spain that have a presence in these social networks with respect to the number of companies in the rest of the Spanish business sector is quite broad. This translates into the greater importance that this hotel sector gives to these tools compared to the rest of the Spanish business sector.
\end{abstract}

Keywords: Hotels, business, online social networks, implementation, internet, ICT.

Dos Algarves: A Multidisciplinary e-Journal, $32-2018$.

ISBN 2182-5580 @ ESGHT - University of the Algarve, Portugal.

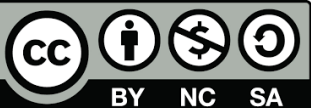

To cite this article: Infante-Moro, A., Infante-Moro, J.C., Martínez-López, F-J., García-Ordaz, M. \& Gallardo-Pérez, P. (2018). La implementación de las redes sociales en el sector hoteleiro español. Dos Algarves: A Multidisciplinary e-Journal, 32, 77-87. doi: 10.18089/DAMeJ.2018.32.5 


\section{Resumo}

Este estudo descreve como o processo de implementação de redes sociais online (Facebook, Twitter ...) tem sido realizado no setor hoteleiro. A análise deste fenómeno foi efetuada através de uma pesquisa e foi considerado o setor empresarial espanhol em geral, como um quadro comparativo. Como resultado, obtivemos que este fenómeno não teve a mesma receção no setor empresarial espanhol como no setor hoteleiro: a diferença entre o número de hotéis em Espanha com presença nas redes sociais e o número de outras empresas também com presença nas redes sociais é muito grande. $O$ que se traduz na maior importância dada pelo setor hoteleiro a essas ferramentas em comparação com o resto do setor empresarial espanhol.

Palavras-chave: Hotéis, empresas, redes sociais, implementação, internet, TIC.

\section{Introducción}

Las redes sociales online generalizadas (Facebook, Twitter, Instagram...) se han convertido en una gran oportunidad para las empresas debido al número de usuarios que poseen, al tiempo que permanecen los usuarios en éstas y a la influencia que ejerce cualquier comentario en estas redes sociales respecto a la compra-venta de cualquier producto, esto ha provocado que a día de hoy sean pocas las empresas que no tienen presencia en ellas o hacen uso de las mismas.

Algo que ha sabido asimilar muy bien el sector hotelero, y ha hecho que sea difícil encontrar actualmente algún hotel que no utilice estas redes sociales online.

Por este motivo y porque este proceso no ha sido de un día para otro, este trabajo estudia la evolución de la implementación de las redes sociales en el sector hotelero español, realizando una comparativa con dicho proceso en el sector empresarial español en general en sus empresas.

Esta investigación parte de un estudio longitudinal que se realiza desde el grupo de investigación GITICE (Grupo de Investigación de las Tecnologías de la Información y las Comunicaciones en la Empresa), que comenzó hace 20 años y que se sigue trabajando en la actualidad. Este estudio realiza macroencuestas sobre Sistemas de Información y Tecnologías en las empresas del sector hotelero español y del sector empresarial español en general con 211 variables cada 10 años: 1991-92, 2001-02 y 2011-12. Y para este artículo se han seleccionado las variables correspondientes a las redes sociales de 2011-12, variables que se agregaron en esa última macroencuesta y que permitirán conocer si esa evolución fue más rápida en las empresas del sector hotelero español que en las empresas del resto del sector empresarial y si han alcanzado el mismo nivel.

Estas variables sobre redes sociales no aparecen en las macroencuestas de 1991-92 y de 2001-02, ya que el origen de estas redes sociales online lo situamos en 2002 (con la aparición de la red social "Friendster") y el origen de las más populares para la presencia de las empresas se sitúa en 2004 y 2006, Facebook y Twitter (respectivamente) (The Cocktail Analysis, 2016; Marketingdirecto.com, 2011).

Así, en el siguiente punto contextualizaremos las redes sociales en las empresas a través de una revisión literaria, en la que se hará un repaso cronológico de los hechos más relevantes del fenómeno de las redes sociales online y se analizarán las oportunidades que éstas crean al sector empresarial y el proceso de implementación de las redes sociales en el sector hotelero. Continuando con la metodología utilizada en el estudio y el análisis de los 
resultados en la fecha concreta seleccionada para hacer la comparativa, concluyendo con las principales diferencias entre ambos sectores en este campo y en su proceso de implementación.

\section{Estado del arte}

Estas redes sociales han tenido una breve pero intensa historia, tal y como recoge Marketingdirecto.com (2011) en el repaso cronológico que hace por los hechos más relevantes de este fenómeno.

1971 - Se envía el primer email entre dos ordenadores, los dos ordenadores se encontraban situados uno al lado del otro.

1978 - Aparece el Bulletin Board System o BBS (Sistema de Tablón de Anuncios), un software para redes de ordenadores que permite a los usuarios conectarse al sistema (a través de internet o a través de una línea telefónica) y utilizar un programa terminal para realizar funciones como descargar software y datos, leer noticias, intercambiar mensajes con otros usuarios, disfrutar de juegos en línea, leer los boletines, etc.

1978 - Se distribuyen las primeras copias de navegadores de internet a través de la plataforma Usenet.

1994 - Se funda GeoCities, una de las primeras Redes Sociales tal y como las conocemos hoy día, permite a los usuarios crear sus propios sitios web y alojarlos en determinados lugares según su contenido.

1995 - TheGlobe.com ofrece a sus usuarios la posibilidad de personalizar sus propias experiencias online publicando su propio contenido e interactuando con otras personas con intereses similares.

1997 - Se lanza AOL Instant Messenger, ofreciendo a los usuarios el chat.

1997 - Aparece Sixdegrees.com, red social que permite la creación de perfiles personales y el listado de amigos.

2000 - La "burbuja de internet" estalla, es el boom del número de ordenadores conectados a Internet.

2002 - Aparece el portal Friendster, pionero en la conexión online de "amigos reales". Alcanza los 3 millones de usuarios en sólo tres meses.

2003 - Aparece MySpace, concebida en sus inicios como un "clon” de Friendster.

2004 - Aparece Facebook, concebida en sus inicios como una plataforma para conectar a estudiantes universitarios. Se implantó por primera vez en la Universidad de Harvard.

2006 - Aparece la red de microblogging Twitter.

2008 - Facebook se convierte en la Red Social líder, adelanta a MySpace en número de visitantes únicos mensuales. 
2011 - Facebook alcanza los 600 millones de usuarios repartidos por todo el mundo, MySpace los 260 millones, Twitter los 190 millones y Friendster llega a los 90 millones.

2016 - Facebook alcanza los 1550 millones de usuarios repartidos por todo el mundo, YouTube los 1000 millones, Google+ los 540 millones, Instagram los 400 millones y Twitter llega a los 320 millones (yourescapefromgto5.com, 2016).

Este repaso cronológico permite ver la dimensión de estas redes sociales, en cuanto al número de usuarios que alcanzan cada una de ellas. Pero a esto, también hay que sumarle el tiempo que permanecen los usuarios en éstas.

Su uso se ha asentado como uno de los principales hábitos de los internautas, el 77,2\% las utilizan a diario en 2015 (muy lejos del 28,6\% del 2008) y más o menos la mitad permanecen en ellas más de media hora al día (Asociación para la Investigación de Medios de Comunicación -[AIMC], 2016). Algo que no ha pasado desapercibido por el mundo empresarial.

Además, los consumidores han empezado a utilizar los comentarios de amigos en redes sociales como una de las principales fuentes de confianza a la hora de buscar información sobre un producto o servicio (Infante-Moro, Zavate \& Infante-Moro, 2015b; Bilgihan, Barreda, Okumus \& Nusair, 2016; The Cocktail Analysis, 2015). El 52,0\% de los consumidores dan mucha o bastante credibilidad a los comentarios de sus amigos en redes sociales sobre un producto o servicio.

Según un estudio realizado en España por la asociación Interactive Advertising Bureau $(I A B, 2016)$ sobre las redes sociales en Internet:

- El 65,0\% de los usuarios declara que las redes sociales influyeron en su compra;

- El 37,0\% de los usuarios busca información en las redes sociales antes de una compra en Internet;

- El 32,0\% de los usuarios realiza comentarios o consultas sobre sus compras en alguna red social;

- Y el 47,0\% de los usuarios declara que los comentarios en redes sociales influyen en su decisión de compra, sólo un $16,0 \%$ niega esta influencia.

Con todo esto, está claro que las empresas han visto en la presencia y uso de estas redes sociales online una ventaja competitiva que ninguna quiere desaprovechar o dejar que otras tomen ventaja gracias a la misma. $\mathrm{Y}$ esta oportunidad ha adquirido gran relevancia en el sector hotelero.

El sector hotelero es uno de los sectores empresariales más competitivos en Internet, lo que ha hecho que todos los hoteles sientan la obligación de estar en estas redes sociales. Aunque es cierto que en un principio esta herramienta trajo mucha controversia.

Muchos hoteles se plantearon su presencia o no presencia en las redes sociales, ya que podrían aparecer comentarios negativos que afectaran en sus futuras reservas (Xiang, Schwartz \& Uysal, 2017; Sánchez \& Fernández, 2017; Martínez María-Dolores, Bernal García \& Mellinas, 2012). Pero como dice el equipo de HotelManager (2015), es difícil pensar que algún hotel no se plantee actualmente su presencia en éstas: es necesario que los hoteles tengan perfiles en las principales redes sociales online (con independencia del tamaño del hotel o del tipo de alojamiento).

Así, se puede dudar de cómo gestionarlas, del personal que se debe hacer responsable de gestionarlas, de las estrategias a seguir, de la elección de estas redes,... pero nunca, de si 
se debe tener presencia en las redes sociales (García, Moraleda \& Curiel, 2017; Zavala \& Estrada, 2016; Mellinas, María-Dolores \& García, 2016; Infante-Moro, Martínez-López \& Infante-Moro, 2015a; Ortiz \& González, 2014).

Todo este proceso no ha sido de un día para otro ni ha afectado de la misma manera a las empresas del sector hotelero español y a las empresas del sector empresarial español en general. Por tanto, este estudio analizará la evolución de la implementación de las redes sociales en las empresas de estos dos sectores en un punto medio de todo este proceso, lo que nos permitirá conocer si este fenómeno ha sido asimilado de la misma manera en el sector hotelero que en el resto del sector empresarial desde un principio y si se ha alcanzado el mismo nivel de importancia en ambos sectores.

\section{Metodología}

En este estudio se utiliza una macroencuesta como instrumento para la captación de datos de las empresas del sector hotelero español y del sector empresarial español en general, la cual se nombró en la introducción (realizada a finales de 2011 y comienzos de 2012). Y de dicha encuesta, se seleccionan cuatro variables que describen la importancia de Internet para estas empresas y cuantifican la presencia de éstas en web y redes sociales, añadiendo si tienen o no implantadas algún tipo de normas sobre la utilización (por parte de los empleados) de estas herramientas; variables cualitativas y cuantitativas que son analizadas posteriormente a través de promedios.

La ficha técnica de la investigación puede verse en la siguiente tabla (ver Tabla 1):

Tabla 1. Ficha técnica de la investigación

\begin{tabular}{|c|c|c|}
\hline & EMPRESAS ESPAÑOLAS & HOTELES \\
\hline ENCUESTA & 2011-12 & 2011-12 \\
\hline $\begin{array}{l}\text { Universo } \\
\text { hipotético }\end{array}$ & $\begin{array}{l}\text { Constituido por las empresas } \\
\text { españolas }\end{array}$ & $\begin{array}{l}\text { Constituido por las empresas } \\
\text { españolas pertenecientes al sector } \\
\text { hotelero }\end{array}$ \\
\hline $\begin{array}{l}\text { Población } \\
\text { objetivo }\end{array}$ & $\begin{array}{l}\text { Empresas españolas, con facturación } \\
\text { superior a } 2 \text { millones de } € \text { anuales }\end{array}$ & $\begin{array}{l}\text { Empresas españolas (sector } \\
\text { hotelero), con facturación superior a } \\
2 \text { millones de } € \text { anuales }\end{array}$ \\
\hline $\begin{array}{l}\text { Población } \\
\text { marco }\end{array}$ & $\begin{array}{l}\text { Base de datos "España } 25.000 \text { " de } \\
\text { Fomento de la Producción }\end{array}$ & $\begin{array}{c}\text { Base de datos “España } 25.000 \text { " de } \\
\text { Fomento de la Producción. Selección } \\
\text { sector hotelero } \\
(417)\end{array}$ \\
\hline Error muestral & $+1-0,049$ & $+\mid-0,09$ \\
\hline $\begin{array}{l}\text { Nivel de } \\
\text { confianza }\end{array}$ & $95,50 \%$ & $95,50 \%$ \\
\hline $\begin{array}{l}\text { Hipótesis } \\
\text { parámetros }\end{array}$ & $\mathrm{Z}=2$ y $\mathrm{P}=\mathrm{Q}=0,5$ & $Z=2$ y $P=Q=0,5$ \\
\hline $\begin{array}{c}\text { Tamaño de la } \\
\text { muestra }\end{array}$ & 410 empresas & 48 hoteles \\
\hline $\begin{array}{l}\text { Procedimiento } \\
\text { de muestreo }\end{array}$ & $\begin{array}{l}\text { Muestreo aleatorio sin reposición, } \\
\text { por números aleatorios }\end{array}$ & $\begin{array}{l}\text { Muestreo aleatorio sin reposición, } \\
\text { por números aleatorios }\end{array}$ \\
\hline $\begin{array}{l}\text { Método } \\
\text { encuesta }\end{array}$ & $\begin{array}{l}\text { Cuestionario por correo postal, } \\
\text { completado por correo electrónico, } \\
\text { teléfono, fax, o visita personal }\end{array}$ & $\begin{array}{l}\text { Cuestionario por correo postal, } \\
\text { completado por correo electrónico, } \\
\text { teléfono, fax, o visita personal }\end{array}$ \\
\hline
\end{tabular}


Tanto para la encuesta llamada HOTELES como para la encuesta EMPRESAS ESPAÑOLAS se utilizan las mismas variables, para poder contrastar los resultados que se obtienen. $Y$ se usa la teoría del muestreo para conseguir datos estadísticamente significativos en ambas; se toma como población de estudio a todas las empresas españolas situadas entre las 25.000 primeras por volumen de facturación recogidas en la base de datos de la revista "Fomento de la Producción" (España 25 000), diferenciándose las empresas del sector hotelero del resto de organizaciones del sector empresarial para la encuesta HOTELES.

En total, en la encuesta HOTELES, en 2011-12 se hicieron 48 encuestas, cuyas respuestas fueron validadas en 2013. La falta de respuesta se evitó con el reenvío de la encuesta y con la sucesiva sustitución por la siguiente empresa en la lista si no contestaba el reenvío. Y las empresas que participaron en la encuesta EMPRESAS ESPAÑOLAS fueron 410 empresas en 2011-12.

\section{Discusión y resultados}

No existe un organismo o entidad que se dedique al estudio global de este fenómeno, sino que son organizaciones públicas y privadas de diferentes países las que elaboran sus propios estudios utilizando sus propias metodologías, provocando que muchos de sus datos publicados no coincidan. Por ese motivo, y por la pronta desactualización de estos datos debido al vertiginoso crecimiento del número de usuarios en cada momento, estamos ante unas herramientas difíciles de cuantificar y en las que sólo debemos quedarnos con conclusiones generalizadas y aproximadas sobre si se utilizan estas herramientas y el porcentaje de empresas que las utilizan.

Las empresas y hoteles deben estar en las redes sociales que los usuarios utilizan para seguir a estas empresas o negocios (Bueno, Meroño \& Piernas, 2017; Torres, 2016), y éstas son Facebook, Twitter e Instagram (IAB, 2016), cuyo orígenes los encontramos en 2004, 2006 y 2010 (respectivamente) (The Cocktail Analysis, 2016; Marketingdirecto.com, 2011).

Desde sus orígenes hasta el día de hoy han pasado pocos años, pero ya en 2011-12 (con el análisis del estado de esa implementación en este punto intermedio) encontrábamos diferencias que nos demostraban que este fenómeno no fue asimilado de la misma manera en el sector hotelero que en el resto del sector empresarial. En este punto, ya observábamos que la importancia que se le daba a Internet en las empresas del sector hotelero era similar a la que se otorgaba en el sector empresarial español. No existían diferencias significativas entre ellas, y ambos sectores eran conscientes de la importancia de Internet en sus mercados, casi la mitad de las empresas españolas y más de la mitad de los hoteles lo consideraban imprescindible y ninguna empresa omitía su importancia (Figura 1). 
Figura 1. Importancia de Internet en el sector empresarial español

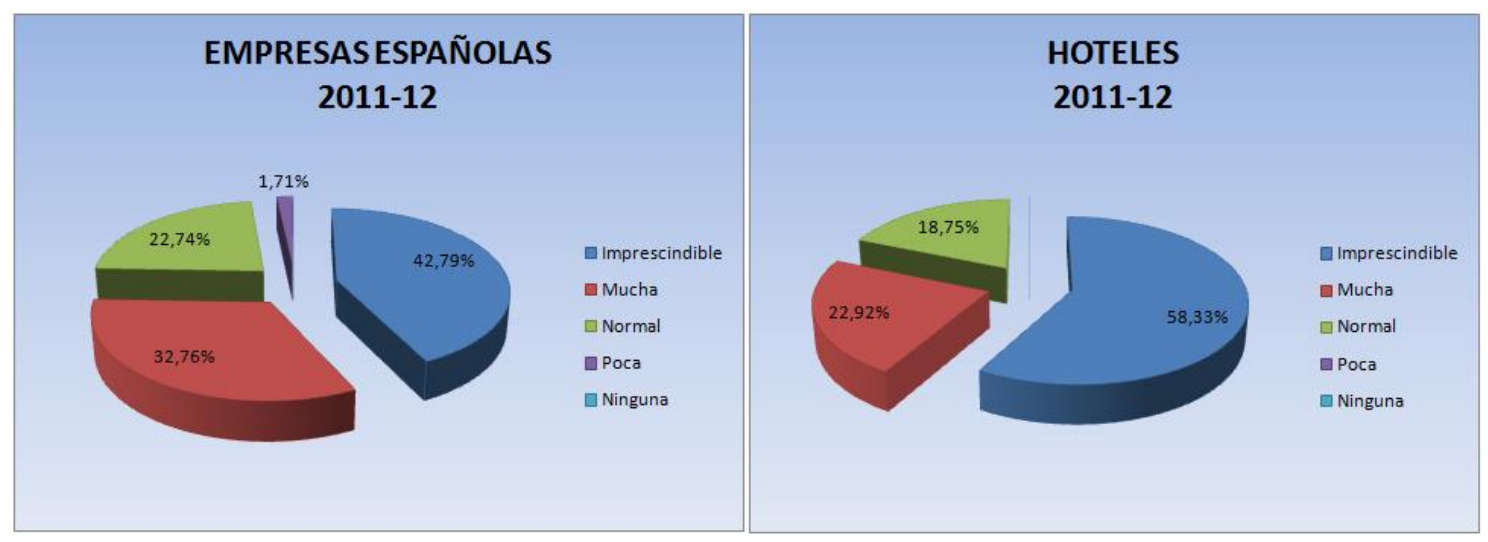

Esa importancia era demostrada en la existencia de páginas web en ambos sectores, donde tampoco existían diferencias significativas y todos los sectores reconocían que si no estaban en Internet, no existían. Su mayor representación era a través de página web, un 96,0\% de las empresas del sector empresarial español y un casi $98,0 \%$ de las empresas de su sector hotelero tenían página web (Figura 2).

Figura 2. Existencia de páginas web en el sector empresarial español

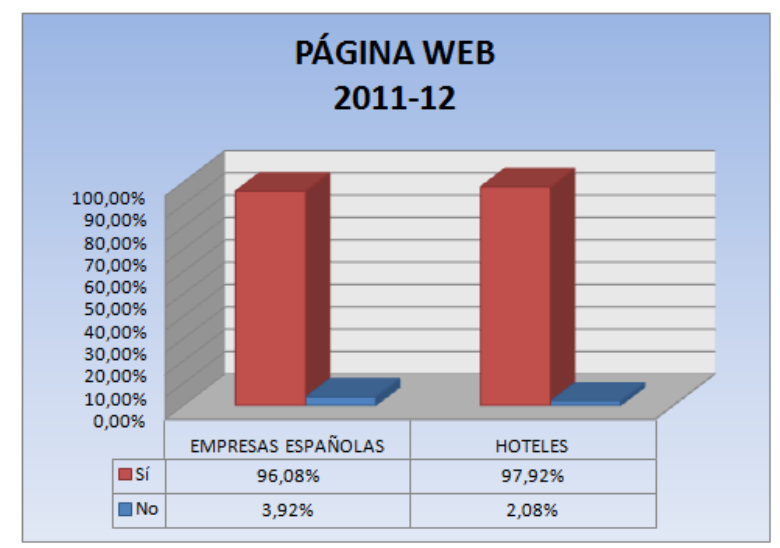

Pero en esos tiempos ya no era suficiente sólo con la existencia de páginas web, millones de personas estaban en las redes sociales y la presencia de estas organizaciones en ellas era necesaria. Esta herramienta aportaba publicidad y ventas si era bien manejada. Aún así, en esta variable sí que encontrábamos ya diferencias significativas entre el sector empresarial español y su sector hotelero (Figura 3).

La presencia en redes sociales era distinta en los dos sectores. Observábamos que todas las empresas del sector hotelero español tenían presencia o la tenían prevista, mientras que un 35,0\% de empresas del sector empresarial no tenían y no la tenían prevista. Esto era una prueba del valor que tenían las redes sociales en el sector hotelero español. 
Figura 3. Presencia de las empresas del sector empresarial español en las redes sociales

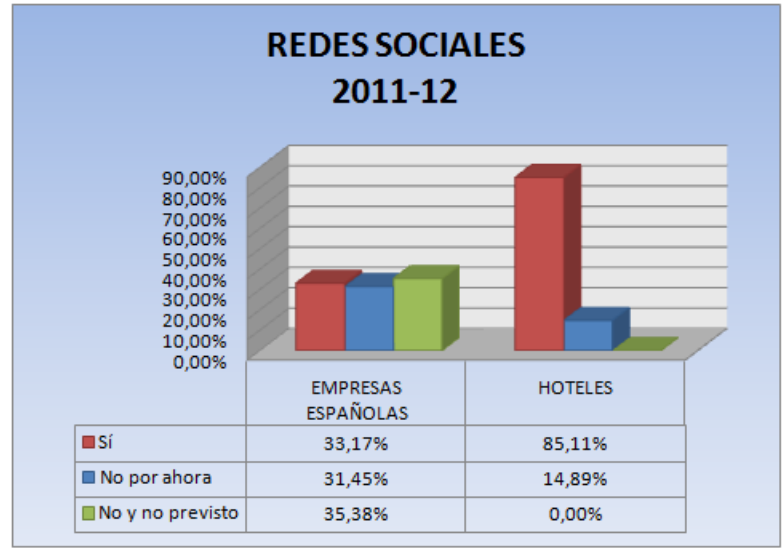

Y a pesar de estas diferencias respecto a la presencia en redes sociales, no existían diferencias significativas entre ambos sectores en la existencia de normas de uso de Internet y redes sociales para los trabajadores (Figura 4). Aunque era cierto que estas organizaciones debían mejorar esos datos, un mal uso de estas herramientas podrían y pueden crear graves problemas a las empresas.

Figura 4. Normas de uso de Internet y redes sociales para los trabajadores en el sector empresarial

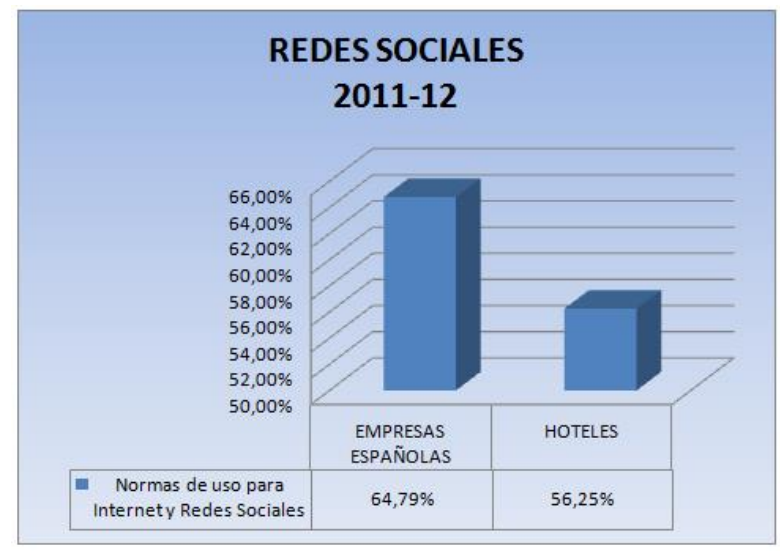

\section{Conclusiones}

Este estudio nos describe cómo ha sido el proceso de implementación de las redes sociales en el sector hotelero, analizando dicho fenómeno en una fecha concreta seleccionada (que es un punto intermedio en todo este proceso que cubre desde sus orígenes hasta el día de hoy) y utilizando como marco comparativo el sector empresarial español en general. Un análisis que nos permite ver que estamos ante dos ámbitos con diferencias significativas en su decisión de tener presencia en redes sociales o no, ya en 2011-12, pero sin diferencias significativas en cuanto al nivel de importancia que le dan Internet, a tener presencia en Internet a través de página web y a la existencia de normas para el uso de Internet y las redes sociales por parte de los trabajadores. 
Por tanto, hablamos de un sector hotelero que ha tenido claro desde un principio que tener presencia en las redes sociales online generalizadas (Facebook, Twitter, Instagram...) supone ser beneficiario de las oportunidades que estas herramientas han traído al sector empresarial. Algo que no ha sucedido en el resto del sector empresarial español en general.

En 2011-12, el 85,11\% de los hoteles en España tenían presencia en las redes sociales y el $14,89 \%$ no tenían presencia pero la tenían prevista. En cambio, estos porcentajes variaban si observábamos al resto del sector empresarial español en general, donde sólo un 33,17\% de empresas tenían presencia en las redes sociales, un 31,45\% no tenían presencia pero la tenían prevista, y existían un $35,38 \%$ de empresas que no tenían presencia y tampoco la tenían prevista.

Pero estas diferencias no sólo son reflejadas en 2011-12, a día de hoy ya encontramos estudios que nos dibujan un sector hotelero español donde el 100,0\% de los hoteles utilizan estas redes sociales (Ortiz \& González, 2014) y un sector empresarial español donde únicamente el 44,0\% de las empresas utilizan estas redes sociales (EFECOM, 2016).

Así, podemos concluir destacando que este fenómeno no ha tenido la misma acogida en el sector hotelero que en el resto del sector empresarial español, ya que tanto en 2011-12 como hoy día la diferencia entre el número de hoteles en España que tienen presencia en estas redes sociales respecto al número de empresas del resto del sector empresarial español es bastante amplia. Lo que se traduce en la mayor importancia que da este sector hotelero a estas herramientas respecto al resto del sector empresarial español.

Este estudio está limitado al sector hotelero español, objeción que puede ser eliminada en estudios futuros extendiéndolo a otros sectores dentro del sector empresarial español para conocer su posterior contextualización en ese mismo marco comparativo, conociendo cómo ha sido este proceso de implementación de las redes sociales en otros sectores.

\section{Referências}

AIMC [Asociación para la Investigación de Medios de Comunicación]. (2016). Navegantes en la Red. Encuesta AIMC a usuarios de Internet. Retrieved from http://www.aimc.es/otros-estudiostrabajos/navegantes-la-red/

Bilgihan, A., Barreda, A., Okumus, F. \& Nusair, K. (2016). Consumer perception of knowledge-sharing in travel-related online social networks. Tourism Management, 52, 287-296. doi.org/10.1016/j.tourman.2015.07.002

Bueno, M. Á. B., Meroño, M. C. P. \& Piernas, J. M. P. (2017). Las redes sociales aplicadas al sector hotelero. International Journal of Scientific Management and Tourism, 3(2), 131-153.

EFECOM (2016). Casi la mitad de las empresas españolas usan redes sociales, en la media UE. Retrieved from http://www.lavanguardia.com/vida/20161214/412618567826/casi-la-mitad-de-lasempresas-espanolas-usan-redes-sociales-en-la-media-ue.html

Fomento de la Producción. (2011). España 25.000. Madrid: Fomento de la Producción.

García, G. C., Moraleda, L. F. \& Curiel, J. D. E. (2017). La interacción de los hoteles con las redes sociales: Un análisis de los hoteles de cuatro estrellas de la comunidad de Madrid (España). Cuadernos de Turismo, 39, 131-148. doi: 10.6018/turismo.39.290471

HotelManager. (2015). Por qué tu hotel debe estar en las redes sociales. Retrieved from http://www.hotelmanager.es/por-que-tu-hotel-debe-estar-en-las-redes-sociales/

IAB [Interactive Advertising Bureau]. (2016). Estudio sobre redes sociales en Internet. Retrieved from http://iabspain.es/wp-content/uploads/IAB_EstudioRedesSociales_2016_completo.pdf

Infante-Moro, A., Martínez-López, F. J. \& Infante-Moro, J. C. (2015a). The extent of computerization in big companies of the spanish hotel sector. Enlightening Tourism. A Pathmaking Journal, 5(2), 126-154. 
Infante-Moro, A., Zavate, A. \& Infante-Moro, J. C. (2015b). The influence/impact of semantic web technologies on social media. International Journal of Information Systems and Software Engineering for Big Companies (IJISEBC), 2(2), 18-30.

Marketingdirecto.com. (2011). Breve historia de las redes sociales. Retrieved from https://www.marketingdirecto.com/digital-general/social-media-marketing/breve-historiade-las-redes-sociales

Martínez María-Dolores, S. M., Bernal García, J. J. \& Mellinas, J. P. (2012). Los hoteles de la región de Murcia ante las redes sociales y la reputación online. Revista de Análisis Turístico, 13, 1-10.

Mellinas, J. P., María-Dolores, S. M. M. \& García, J. J. B. (2016). El uso de redes sociales por los hoteles como indicativo de gestión eficiente. Tourism \& Management Studies, 12(2), 78-83. doi: 10.18089/tms.2016.12209

Ortiz, L. \& González, R. (2014). Las redes sociales como herramienta de mejora de la experiencia turística: Una aplicación al sector hotelero. Revista Iberoamericana de Turismo - RITUR, 4(1), 16-34.

Sánchez, D. H. M. \& Fernández, J. M. (2017). Análisis de comentarios en redes sociales para mejorar la reputación online hotelera. Turismo y Sociedad, 20, 169-190. doi.org/10.18601/01207555.n20.09

The Cocktail Analysis. (2015). VII Observatorio de redes sociales. Retrieved from http://es.slideshare.net/arena es/vii-observatorio-de-redes-sociales

The Cocktail Analysis. (2016). VIII Observatorio de redes sociales. Retrieved from http://es.slideshare.net/arena_es/observatorio-rrss-viii-ola-70186506

Torres, A. (2016). Las 5 mejores redes sociales para tu hotel. Retrieved from http://www.anatorresdamas.com/5-mejores-redes-sociales-para-tu-hotel/

Xiang, Z., Schwartz, Z. \& Uysal, M. (2017). Market intelligence: Social media analytics and hotel online reviews. In Analytics in Smart Tourism Design (pp. 281-295). Cham, Suiza: Springer.

yourescapefromgt05.com (2016). Usuarios mensuales de las redes sociales en 2016. Retrieved from http://yourescapefrom9to5.com/social-media-monthly-active-users-infographic

Zavala, A. M. M. \& Estrada, I. C. (2016). Redes sociales: Herramienta de marketing internacional en el sector hotelero. El Periplo Sustentable, 31 [online]. Disponivel em: http://www.redalyc.org/jatsRepo/1934/193449985013/index.html

ALFONSO INFANTE MORO es doctor en Ciencias Económicas y Empresariales por la Universidad de Huelva, España. Miembro del Grupo de Investigación GITICE (www.gitice.com) y profesor del Departamento de Economía Financiera, Contabilidad y Dirección de Operaciones de la Universidad de Huelva, España. Endereço institucional: Universidad de Huelva, Facultad de Ciencias Empresariales, Departamento de Economía Financiera, Contabilidad y Dirección de Operaciones, Campus de «La Merced», Plaza de la Merced, 11. 21007. Huelva (España).

JUAN CARLOS INFANTE MORO es doctor en Dirección de Empresas Turísticas por la Universidad de Huelva, España. Miembro del Grupo de Investigación GITICE (www.gitice.com) y profesor del Departamento de Economía Financiera, Contabilidad y Dirección de Operaciones de la Universidad de Huelva, España. Endereço institucional: Universidad de Huelva, Facultad de Ciencias Empresariales, Departamento de Economía Financiera, Contabilidad y Dirección de Operaciones, Campus de «La Merced», Plaza de la Merced, 11. 21007. Huelva (España).

FRANCISCO JOSÉ MARTÍNEZ LÓPEZ es doctor en Ciencias Económicas y Empresariales. Miembro del Grupo de Investigación GITICE (www.gitice.com) y profesor del Departamento de Economía Financiera, Contabilidad y Dirección de Operaciones de la Universidad de Huelva, España. Endereço institucional: Universidad de Huelva, Facultad de Ciencias Empresariales, Departamento de Economía Financiera, Contabilidad y Dirección de Operaciones, Campus de «La Merced», Plaza de la Merced, 11. 21007. Huelva (España).

MERCEDES GARCÍA ORDAZ es doctora en Ciencias Económicas y Empresariales. Miembro del Grupo de Investigación GITICE (www.gitice.com) y profesora del Departamento de Economía Financiera, Contabilidad y Dirección de Operaciones de la Universidad de Huelva, España. Endereço institucional: Universidad de Huelva, Facultad de Ciencias Empresariales, Departamento de Economía Financiera, 
Contabilidad y Dirección de Operaciones, Campus de «La Merced», Plaza de la Merced, 11. 21007. Huelva (España).

JULIA GALLARDO PÉREZ es licenciada en Ciencias Económicas y Empresariales por la Universidad de Huelva, España. Endereço institucional: Universidad de Huelva, Facultad de Ciencias Empresariales, Departamento de Economía Financiera, Contabilidad y Dirección de Operaciones, Campus de «La Merced», Plaza de la Merced, 11. 21007. Huelva (España).

Submetido em 17 outubro 2017

Aceite em 24 março 2018 